\title{
Lyapunov Exponents of One Dimensional Chaotic Dynamical Systems via a General Piecewise Spline Maximum Entropy Method
}

\author{
Md Shafiqul Islam ${ }^{1 *}$ \\ ${ }^{1}$ School of Mathematical and Computational Sciences, University of Prince Edward Island, Charlottetown, Canada, \\ *Corresponding author E-mail: sislam@ upei.ca
}

\begin{abstract}
Article Info
Keywords: Dynamical systems, General piecewise spline method, Invariant measure and invariant density, Lyapunov exponents, Maximum entropy optimization method

2010 AMS: 37A05, 37A35,37E05

Received: 04 July 2019

Accepted: 16 November 2019

Available online: 20 December 2019
\end{abstract}

Abstract

\section{Introduction}

In a chaotic dynamical system, inaccuracies in specifying the initial state of the system are rapidly amplified in time and therefore, it is impossible to predict the long term system state. If nearby trajectories of a dynamical system diverges exponentially then the dynamical system possesses chaotic behaviour [1]-[3]. The rate of increase of perturbations of initial conditions is described by the Lyapunov exponent [4]. The Lyapunov exponent of a dynamical system classifies the dynamics of the system. A dynamical system with positive Lyapunov exponent exhibits chaotic nature of the system. Let $\tau:[0,1] \rightarrow[0,1]$ be a measure preserving deterministic dynamical system (map) and $\mu$ be a $\tau$-invariant measure on the probability space $[0,1]$. For any $x \in[0,1]$, the Lyapunov exponent $h(x)$ of the trajectory of $x$ is defined as (see, for example, [5])

$$
\begin{aligned}
h(x) & =\lim _{n \rightarrow \infty} \ln \left(\mid\left(\left(\tau^{n}(x)\right)^{\prime} \mid\right)^{\frac{1}{n}}\right. \\
& =\lim _{n \rightarrow \infty} \frac{1}{n}\left(\ln \left|\tau^{\prime}(x)\right|+\ln \mid \tau^{\prime}\left(\tau(x)|+\cdots+\ln | \tau^{\prime}\left(\tau^{n-1}(x) \mid\right)\right.\right. \\
& =\lim _{n \rightarrow \infty} \frac{1}{n} \sum_{i=0}^{n-1} \ln \left|\tau^{\prime}\left(\tau^{i}(x)\right)\right|
\end{aligned}
$$

provided the limit exists. If the function $\ln \left(\tau^{\prime}(x)\right)$ is integrable and $\tau$ is ergodic, then the Birkhoff Ergodic Theorem [6] gaurantees that for almost all $x \in[0,1]$ the above limit exists, it is a constant (say, $l$ ) and if $f$ is the density of the invariant measure $d \mu(x)=f(x) d \lambda(x)$, then the Lyapunov exponent

$$
l=\int_{0}^{1} \ln \left|\tau^{\prime}(x)\right| d \mu(x)=\int_{0}^{1} \ln \left|\tau^{\prime}(x)\right| f(x) d \lambda(x),
$$

where $\lambda$ is the underlying Lebesgue measure on $[0,1]$. Therefore, if the invariant measure $\mu$ or the density function $f$ is known, then one can calculate the Lyapunov exponent of $\tau$ in (1.1). Unfortunately, except for some cases, the analytical formula of the invariant measure 
$\mu$ or the density function $f$ for most of the deterministic maps is not known. Therefore, the computation of Lyapunov exponent havily rely on numerical approximation of invariant measures or density functions of the corresponding deterministic maps. Computations of Lyapunov exponents for deterministic dynamical systems are well studied by many researchers. A spatial average estimation method for Lyapunov exponents of deterministic maps is presented in [7] by G. Froyland. A numerical scheme is described in [8] for the $n$ Lyapunov exponents of an $n$-dimensional unknown dynamical system. In [4], A. Boyarsky presented a matrix method for the approximation of Lyapunov exponents and invariant measures. Lyapunov exponents from observed time series is studied in [9]. For Jacobian-based estimates of Lyapunov exponents from data, convergence rates and data requirements are studied in [10].

In the context of information theory, the principle of maximum entropy [11] was introduced by E. T. Jaynes [12] in 1957. Since then, mathematicians, physicists and engineers have widely used the maximum entropy method in many different directions for solving problems in mathematics, mathematical physics and other related branches of Science and Engineering. A maximum entropy method was described by Lawrence R. Mead and N. Papanicolaou in [11] for solving moment problems. For finite approximation of the Frobenius-Perron operator of deterministic dynamical systems the maximum entropy methods were described by many authors [13]-[18] and [19]. In [20], C. Bose and R. Murray presented dynamical conditions for convergence of a maximum entropy method for Frobenius-Perron operator equations. For approximation of invariant measures for position dependent random maps we have described maximum entropy methods in [21, 22]. An iterative maximum entropy method is presented in [23] for Lyapunov exponents and invariant densities for deterministic chaotic maps. In this paper, we compute Lyapunov exponents via a general piecewise spline maximum entropy method. Moreover, we compare our results between piecewise linear, piecewise quadratic and piecewise cubic maximum entropy methods for the computation of Lyapunov exponents.

In Section 2, we present a general piecewise spline maximum entropy method for approximation of invariant measures of deterministic dynamical systems. Moreover, we present convergence analysis of maximum entropy method. In Section 3, we present calculations of Lyapunov exponents of deterministic dynamical systems using general piecewise spline maximum entropy method. We present two numerical examples with a comparison between piecewise linear, piecewise quadratic and piecewise cubic maximum entropy method.

\section{A general piecewise spline maximum entropy optimization method for invariant measures of deterministic chaotic dynamical systems}

Let $(I=[0,1], \mathscr{B}, \lambda)$ be a measure space, where $\mathscr{B}$ is a $\sigma$-algebra on $I=[0,1]$ and $\lambda$ is the Lebesgue measure on $\mathscr{B}$. Let $\tau: I \rightarrow I$ be a deterministic map such that $\tau$ has a unique absolutely continuous invariant measure $\mu^{*}$ with density $f^{*}$. Using (1.2) one can find the actual Lyapunov exponent $L$ for $\tau$. In this section, first we revisit a general piecewise spline maximum entropy optimization method for the approximation $f_{n}$ of the density function $f^{*}$. In the next section, using the approximate density $f_{n}$ we compute an approximate Lyapunov exponent $l_{n}$. We also present the convergence analysis of the general piecewise maximum entropy method. We closely follow [6] and [13].

Let

$$
\cdots<x_{-2}<x_{-1}<x_{0}=0<x_{1}<x_{2}<\ldots
$$

be an infinite set of nodes on the real line. The B-splines of degree $k$ are defined recursively as follows:

$$
B_{i}^{0}(x)=\left\{\begin{array}{ll}
1, & x \in\left[x_{i}, x_{i+1}\right), \\
0, & x \notin\left[x_{i}, x_{i+1}\right),
\end{array}, i \in\{\ldots,-2,-1,0,1,2, \ldots\}\right.
$$

and

$$
B_{i}^{k}(x)=\frac{x-x_{i}}{x_{i+k}-x_{i}} B_{i}^{k-1}(x)+\frac{x_{i+k+1}-x}{x_{i+k+1}-x_{i+1}} B_{i+1}^{k-1}(x), i \in\{\ldots,-2,-1,0,1,2, \ldots\}
$$

Each $B_{i}^{k}(x)$ is a piecewise polynomial of degree $k$ and $B_{i}^{k}(x)$ are called $B$-splines of degree $k$. The family $\left\{B_{i}^{k}\right\}$ of $B$-splines satisfies the following properties (see Proposition 1 in [13]):

Properties of $B$-splines:

1. If $x \notin\left[x_{i}, x_{i+k+1}\right)$, then $B_{i}^{k}(x)=0$;

2. If $x \in\left(x_{i}, x_{i+k+1}\right)$, then $B_{i}^{k}(x)>0$;

3. $\sum_{i} B_{i}^{k}(x)=1$ for all $x$;

4. For fixed $k$, the set $\left.\left\{\left.B_{i}^{k}\right|_{\left[x_{o}, x_{n}\right]}\right\}:-k \leq i \leq n-1\right\}$ of functions constitute a basis for the space $\Delta_{n}^{k}$ consisting of all functions in $C^{k-1}\left[x_{0}, x_{n}\right]$ which are piecewise polynomials of degree $\leq k$ on the $n$ subintervals $\left[x_{0}, x_{1}\right],\left[x_{1}, x_{2}\right], \ldots,\left[x_{n-1}, x_{n}\right]$.

Let $\tau: I \rightarrow I$ be a deterministic map such that $\tau$ has a unique absolutely continuous invariant measure $\mu^{*}$ with density $f^{*}$. A particular choice of $\tau$ is a Lasota - Yorke map described in [24]. Note that the invariant density $f^{*}$ of the unique acim $\mu^{*}$ is the fixed point of the Frobenius-Perron operator $P_{T}$. In the following, we describe a general spline maximum entropy approximation scheme for $f^{*}$. 
Let $\mathscr{D}$ be the set of all densities, that is,

$$
\mathscr{D}=\left\{f \in L^{1}(0,1) \text { such that } \mathrm{f} \geq 0 \text { and }\|\mathrm{f}\|_{1}=\int_{0}^{1} \mathrm{f}(\mathrm{x}) \mathrm{d} \lambda(\mathrm{x})=1\right\} .
$$

The Boltzmann entropy [6] of $f \geq 0$ is defined by

$$
H(f)=-\int_{I} f(x) \log f(x) d \lambda(x) .
$$

For properties of $\mathrm{H}$ see [6]. Using the Gibbs inequality

$$
u-u \log u \leq v-u \log v, u, v \geq 0,
$$

it can be shown that

$$
\int_{I} f(x) \log f(x) d \lambda(x) \geq \int_{I} f(x) \log g(x) d \lambda(x) \forall f, g \in \mathscr{D} .
$$

The above inequality in (2.2) leads to the following optimization problem [14]:

$$
\max H(f) \text { such that } \mathrm{f} \in \mathscr{D} \text { and } \int_{\mathrm{I}} \mathrm{f}(\mathrm{x}) \mathrm{g}_{\mathrm{n}}(\mathrm{x}) \mathrm{d} \lambda(\mathrm{x})=\mathrm{m}_{\mathrm{n}}, 1 \leq \mathrm{n} \leq \mathrm{N},
$$

where $m_{1}, m_{2}, \cdots, m_{N}$ are given moments of the unknown density with respect to the moment function $\left\{g_{1}, g_{2}, \ldots, g_{N}\right\} \subset L^{\infty}(I)$, respectively

Proposition 2.1. [6] Suppose that $a_{1}, a_{2}, \cdots, a_{N}$ are real numbers such that the function

$$
f_{N}(x)=\frac{e^{\sum_{n=1}^{N} a_{n} g_{n}(x)}}{\int_{0}^{1} e^{\sum_{n=1}^{N} a_{n} g_{n}(x)} d \lambda(x)}
$$

satisfies the constraints in (2.3), that is,

$$
\frac{\int_{0}^{1} g_{i}(x) e^{\sum_{n=1}^{N} a_{n} g_{n}(x)} d \lambda(x)}{\int_{0}^{1} e^{\sum_{n=1}^{N} a_{n} g_{n}(x)} d \lambda(x)}=m_{i}, i=1,2, \ldots, N .
$$

Then $f_{N}$ is a unique solution of the maximum entropy problem (2.3).

Proof: See [6].

Let $\mathscr{P}^{(n)}=\left\{I_{1}, I_{2}, \ldots, I_{n}\right\}$ partition of $[0,1]$ into $n$ equal subintervals, where $I_{i}=\left[b_{i-1}, b_{i}\right]$,

$b_{i}=i h, i=1,2, \ldots, n, b_{0}=0, b_{n}=1, h=\frac{1}{n}$. Without loss of generality and for convenience, we consider $n=2^{s}=l \cdot q$, where $l$ is a positive even integer and $q$ is the number of the sub-intervals of the partition $\mathscr{J}$ on which the maps $\tau$ is piecewise one-to-one and monotonic. Consider $2 k$ additional nodes: $b_{-j}=-j h, b_{n+j}=(n+j) h, j=1,2, \ldots, k$. These nodes are needed to express all the involved $B$-splines for the state space $I=[0,1]$. Moreover, for fixed $k$, the set

$$
\left.\left\{\left.B_{i}^{k}\right|_{[0,1]}\right\}: i=-k,-k+1, \ldots, 0,1,2, \ldots, n-1\right\}
$$

of functions constitute a basis for the space $\Delta_{n}^{k}$ consisting of all functions in $C^{k-1}[0,1]$ which are piecewise polynomials of degree $\leq k$ on the $n$ subintervals $\left[b_{0}, b_{1}\right],\left[b_{1}, b_{2}\right], \ldots,\left[b_{n-1}, b_{n}\right]$ of $I=[0,1] . \Delta_{n}^{k}$ has dimension $n+k$ and $B_{k}^{k}, B_{-k+1}^{k}, \ldots, B_{0}^{k}, B_{1}^{k}, \ldots, B_{n-1}^{k}$ are elements of the basis for $\Delta_{n}^{k}$.

Let $f^{*}$ be an unique density function of the acim $\mu^{*}$ for the map $\tau$. Then the moments of $f^{*}$ with respect to $B$-spline $B_{i}^{k}, i=-k,-k+$ $1, \ldots, 0,1,2, \ldots, n-1$ is

$$
m_{i}=\int_{0}^{1} f^{*}(x) B_{i}^{k}(x) d \lambda(x)
$$

Proposition 2.2.

$$
\sum_{i=-k}^{n-1} m_{i}=1
$$

Proof:

$$
\begin{aligned}
\sum_{i=-k}^{n-1} m_{i} & =\sum_{i=-k}^{n-1} \int_{0}^{1} f^{*}(x) B_{i}^{k}(x) d \lambda(x)=\int_{0}^{1} f^{*}(x) \sum_{i=-k}^{n-1} B_{i}^{k}(x) d \lambda(x) \\
& =\int_{0}^{1} f^{*}(x) d \lambda(x)=1,
\end{aligned}
$$

since $\sum_{i=-k}^{n-1} B_{i}^{k}(x)=1$, by Property (iii). 
Proposition 2.3. Suppose that $m_{-k}, m_{-k+1}, \ldots, m_{0}, m_{1}, \ldots, m_{n-1}$ are moments defined in (2.4) of the probability density function $f^{*}$ of the map $\tau$. If $a_{-k}, a_{-k+1}, \ldots, a_{0}, a_{1}, \ldots a_{n-1}$ are constants which satisfy

$$
\int_{0}^{1} B_{i}^{k}(x) e^{\sum_{i=-k}^{n-1} a_{i} B_{i}^{k}(x)} d \lambda(x)=m_{i}, i=-k,-k+1, \ldots, 0,1, \ldots, n-1
$$

then

$$
f_{n}^{k}(x)=e^{\sum_{i=-k}^{n-1} a_{i} B_{i}^{k}(x)}
$$

is a density.

Proof.

$$
\begin{aligned}
\int_{0}^{1} f_{n}^{k}(x) d \lambda(x) & =\int_{0}^{1} e^{\sum_{i=-k}^{n-1} a_{i} B_{i}^{k}(x)} d \lambda(x) \\
& =\int_{0}^{1} e^{\sum_{i=-k}^{n-1} a_{i} B_{i}^{k}(x)} \sum_{i=-k}^{n-1} B_{i}^{k}(x) d \lambda(x) \\
& =\sum_{i=-k}^{n-1} \int_{0}^{1} B_{i}^{k}(x) e^{\sum_{i=-k}^{n-1} a_{i} B_{i}^{k}(x)} d \lambda(x) \\
& =\sum_{i=-k}^{n-1} m_{i}=1
\end{aligned}
$$

If we solve the maximum entropy problem

$$
\max H(f) \text { such that } f \in \mathscr{D} \text { and } \int_{I} f(x) B_{i}^{k}(x) d \lambda(x)=m_{i},-k \leq i \leq n-1,
$$

then $f_{n}^{k}(x)=e^{\sum_{i=-k}^{n-1} a_{i} B_{i}^{k}(x)}$ is a solution of (2.6) such that there exists constants $a_{-k}, a_{-k+1}, \ldots, a_{0}, a_{1}, a_{2}, \ldots, a_{n-1}$ satisfying

$$
\int_{0}^{1} B_{i}^{k}(x) e^{\sum_{i=-k}^{n-1} a_{i} B_{i}^{k}(x)} d \lambda(x)=m_{i}, i=-k,-k+1, \ldots, 0,1, \ldots, n-1 .
$$

Now, using the Birkhoff's Ergodic Theorem (see below), we describe a method for estimating the moments $m_{-k}, m_{-k+1}, \ldots, m_{0}, m_{1}, \ldots, m_{n-1}$ in (2.6) for the unknown invariant density $f^{*}$ of a map $\tau$ :

Theorem 2.4. If $\mu^{*}$ is $\tau$-invariant, $\mu^{*}$ is absolutely continuous and unique among absolutely continuous invariant measures, $P_{\tau}$ satisfies

$$
\left\|P_{\tau}^{n} f\right\|_{B V} \leq A\|f\|_{B V}+B\|f\|_{1},
$$

where $A>0$ and $B>0$ are constants defined in [24]. Then for $\mu^{*}$ almost every $x$ with probability 1 ,

$$
\frac{1}{M} \sum_{i=0}^{M-1} f\left(\tau^{i}(x)\right) \rightarrow \mu^{*}(f)
$$

for any $f \in L^{1}([0,1])$. Moreover, if $\left[0,1\right.$ is a probability space (that is, $\mu^{*}$ is a probability measure on $\left.[0,1]\right)$ and $\tau$ is ergodic, then there exists a function $\hat{f} \in L^{1}([0,1])$ such that

$$
\lim _{M \rightarrow \infty} \frac{1}{M} \sum_{i=0}^{M-1} f\left(\tau^{i}(x)\right)=\hat{f}(x), \forall x \in[0,1] \mu^{*}-\text { a.e. }
$$

and $\hat{f}$ is constant and $\hat{f}=\int_{0}^{1} f(x) d \mu^{*}(x)$.

Note that $f^{*}$ is the density of the map $\tau$ with respect to the acim $\mu^{*}$. Thus, $d \mu^{*}(x)=f^{*}(x) d \lambda(x)$. If we replace $f$ by $B_{i}^{k}, i=-k,-k+$ $1, \ldots, 0,1,2, \ldots, n-1$ and $\hat{f}$ by $m_{i}$ then from Equation (2.7) we obtain

$$
m_{i}=\lim _{M \rightarrow \infty} \frac{1}{M} \sum_{j=0}^{M-1} B_{i}^{k}\left(\tau^{j}(x)\right), \forall x \in[0,1] \text { a.e. }, i=k,-k+1, \ldots, 0,1, \ldots, n-1 .
$$

For large $M$, define

$$
\hat{m}_{i}=\frac{1}{M} \sum_{j=0}^{M-1} B_{i}\left(\tau^{j}(x)\right), \forall x \in[0,1] \text { a.e. }, i=k,-k+1, \ldots, 0,1, \ldots, n-1 .
$$

Note that the choice of $x$ almost surely doesn't matter asymptotically. Now, we consider the following normalized approximation for moments (for convenience, we denote them by $m_{i}$.):

$$
m_{i} \approx \frac{\hat{m}_{i}}{\sum_{i=-k}^{n-1} \hat{m}_{i}}, i=k,-k+1, \ldots, 0,1, \ldots, n-1 .
$$


Note that if $a_{-k}, a_{-k+1}, \ldots, a_{0}, a_{2}, \ldots, a_{n-1}$ satisfy (2.5) then $a_{-k}, a_{-k+1}, \ldots, a_{0}, a_{2}, \ldots, a_{n-1}$ also satisfy

$$
\frac{\int_{0}^{1} B_{i}^{k}(x) e^{\sum_{i=-k}^{n-1} a_{i} B_{i}^{k}(x)} d \lambda(x)}{\int_{0}^{1} e^{\sum_{i=-k}^{n-1} a_{i} B_{i}^{k}(x)} d \lambda(x)}=m_{i}, i=-k,-k+1, \ldots, 0,1,2, \ldots, n-1 .
$$

The nonlinear equations in (2.5) form the following system of $n+k$ nonlinear equations:

$$
G(a)=0,
$$

where $G: \mathbb{R}^{n+k} \rightarrow \mathbb{R}^{n+k}$ defined by

$$
\begin{array}{r}
G_{i}\left(a_{-k}, a_{-k+1}, \ldots, a_{0}, a_{1}, \ldots, a_{n-1}\right)=\int_{0}^{1} B_{i}^{k}(x) e^{\sum_{i=-k}^{n-1} a_{i} \phi_{i}^{k}(x)} d \lambda(x)-m_{i}, \\
i=-k,-k+1, \ldots, 0,1, \ldots, n-1 .
\end{array}
$$

The Jacobian matrix $G^{\prime}=\left(g_{i, j}^{\prime}\right)_{-k \leq i, j \leq n-1}$ of $G$ is defined by

$$
g_{i, j}^{\prime}=\frac{\partial G_{i}}{\partial a_{j}}=\int_{0}^{1} B_{i}^{k}(x) e^{\sum_{i=-k}^{n-1} a_{i} B_{i}^{k}(x)} B_{j}^{k}(x) d \lambda(x), i, j=-k,-k+1, \ldots, 0,1, \ldots, n-1 .
$$

Proposition 2.5. The system (2.5) has a unique solution $a=\left(a_{-k}, a_{-k+1}, \ldots, a_{0}, a_{1}, \ldots, a_{n-1}\right)$.

Proof. Since support of $B_{i}^{k}$ and $B_{j}^{k}$ are disjoint for $|i-j|>k$, it is clear that Jacobian matrix $G^{\prime}$ of $G$ is symmetric. Let

$$
\beta=\left(\beta_{-k}, \beta_{-k+1}, \ldots, \beta_{0}, \beta_{1}, \ldots, \beta_{n-1}\right) \in \mathbb{R}^{n+k} .
$$

Then,

$$
\begin{aligned}
\beta G^{\prime} \beta^{T} & =\sum_{i=-k}^{n-1} \sum_{j=-k}^{n-1} \beta_{i} \beta_{j} \int_{0}^{1} B_{i}^{k}(x) e^{\sum_{i=-k}^{n-1} a_{i} B_{i}^{k}(x)} B_{j}^{k}(x) d \lambda(x) \\
& =\int_{0}^{1} \sum_{i=-k}^{n-1} \sum_{j=-k}^{n-1} \beta_{i} B_{i}^{k}(x) e^{\sum_{i=-k}^{n-1} a_{i} B_{i}^{k}(x)} B_{j}^{k}(x) \beta_{j} d \lambda(x) \\
& =\int_{0}^{1}\left(\sum_{i=-k}^{n-1} \beta_{i} B_{i}^{k}(x)\right) e^{\sum_{i=-k}^{n-1} a_{i} B_{i}^{k}(x)}\left(\sum_{j=-k}^{n-1} B_{j}^{k}(x) \beta_{j}\right) d \lambda(x)>0 .
\end{aligned}
$$

Thus, $G^{\prime}$ is positive definite. Let

$$
g\left(a_{-k}, a_{-k+1}, \ldots, a_{0}, a_{1}, \ldots, a_{n-1}\right)=\int_{0}^{1} e^{\sum_{i=-k}^{n-1} a_{i} B_{i}^{k}(x)} d \lambda(x)-\sum_{i=-k}^{n-1} a_{i} m_{i} .
$$

Now, consider the following global minimization problem:

$$
\min _{\left(a_{-k}, a_{-k+1}, \ldots, a_{0}, a_{1}, \ldots, a_{n-1}\right) \in \mathbb{R}^{n+k}} g\left(a_{-k}, a_{-k+1}, \ldots, a_{0}, a_{1}, \ldots, a_{n-1}\right)
$$

It can be shown that the system (2.5) of equations is the gradient equation of the global minimization problem (2.9). Since $G^{\prime}$ is positive definite, the objective function $g$ is strictly convex on $\mathbb{R}^{n+k}$. Moreover, $g\left(a_{-k}, a_{-k+1}, \ldots, a_{0}, a_{1}, \ldots, a_{n-1}\right)$ is coercive on its domain. Thus, the above global unconditioned convex programming problem (2.9) has a unique solution and therefore, the system (2.5) has a unique solution.

Algorithm: Choose $n$ and $k$. Calculate the moments $m_{i}, i=-k,-k+1, \ldots, 0,1 \ldots, n-1$ using $(2.8)$ and use these moments $m_{i}, i=$ $-k,-k+1, \ldots, 0,1 \ldots, n-1$ to find $a=\left(a_{-k}, a_{-k+1}, \ldots, a_{0}, a_{1}, \ldots, a_{n-1}\right)$. Then the solution of the the maximum moment problem (2.6) is

$$
f_{n}^{k}(x)=e^{\sum_{i=-k}^{n-1} a_{i} B_{i}^{k}(x)}
$$

which is an approximation of the density function $f^{*}$ of the acim $\mu^{*}$ for the map $\tau$.

For convergence analysis, we also assume that the unique invariant measure $f^{*}$ of $\tau$ is used in Equation (2.4) for the calculations of moments $m_{i}, i=-k,-k+1, \ldots, 0,1, \ldots, n-1$. Our convergence analysis is based on the following general convergence theory for moment problem developed by Borwein and Lewis in [25].

Let $X$ be a locally convex topological vector space with nested sequence of compact subsets $\left\{G_{n}\right\}$. Let $W: X \rightarrow[-\infty, \infty)$ be a functional with compact level sets. Let $g_{n}$ be an optimal solution of $\max \left\{W(h): h \in G_{n}\right\}$ and $g_{\infty}$ be the unique optimal solution of the limiting problem $\max \left\{W(h): h \in \cap_{n=1}^{\infty} G_{n}\right\}$ with $W\left(g_{\infty}\right)>-\infty$. It was proved in [25] that $\lim _{n \rightarrow \infty} g_{n}=g_{\infty}$ under the topology of $X$ and $\lim _{n \rightarrow \infty} W\left(g_{n}\right)=$ $W\left(g_{\infty}\right)$. For our B-spline maximum entropy method of degree $k$, we partition the interval $[0,1]$ in the following special way so that the feasible sets are monotonically decreasing. First we divide $[0,1]$ into $k+1$ equal subintervals. Then we divide one subinterval of the current partition into $k+1$ equal parts at each step in succession. Thus the corresponding $n+k$-dimensional spaces $\Delta_{n}^{k}$ of spline functions of degree $k$ are monotonically increasing, which guarantees that the feasible sets of the maximum entropy method are monotonically decreasing. Furthermore, by property of the Boltzmann entropy in the previous section, these feasible sets are weakly compact in $L^{1}(0,1)$. Since $L^{1}(0,1)$ is a locally convex topological vector space in the weak topology, the above weak convergence implies the weak convergence of our method. Thus, we have the following theorem: 
Theorem 2.6. Let $\tau: I \rightarrow I$ be a deterministic dynamical system with the unique fixed point $f^{*}$ of the Frobenius - Perron operator $P_{\tau}$ satisfying $H\left(f^{*}\right)>-\infty$ and $f_{n}$ be the sequence of solutions in (2.10). Then $\lim _{n \rightarrow \infty} f_{n}=f^{*}$ weakly and $\lim _{n \rightarrow \infty} H\left(f_{n}\right)=H\left(f^{*}\right)$.

Note that a functional $W$ on a normed space $X$ into $[-\infty, \infty)$ is called a Kadec if $x_{n} \rightarrow x$ weakly and $W\left(x_{n}\right) \rightarrow W(x)$ imply $x_{n} \rightarrow x$ strongly It can be shown that Boltzmann entropy functional $H$ in (2.1) is Kadec (see [25]). Thus, we have the following strong convergence result.

Theorem 2.7. Let $\tau: I \rightarrow I$ be a deterministic dynamical system with the unique fixed point $f^{*}$ of the Frobenius - Perron operator $P_{\tau}$ satisfying $H\left(f^{*}\right)>-\infty$ and $f_{n}$ be the sequence of solutions in (2.10). Then $\lim _{n \rightarrow \infty}\left\|f_{n}-f^{*}\right\|_{1}=0$.

\section{Numerical examples for Lyapunov exponents and invariant measures of one dimensional chaotic maps via piecewise spline maximum entropy method}

In this section, we give two the results from two numerical examples to illustrate our method. Our first example uses the well-known logistic map for which the density function $f *$ of the acim is known and hence hence the analytical Lyapunov exponent $l$ is also known. The second example uses a piecewise polynomial mapping whose corresponding density function is not known. For both of these examples we apply our piecewise spline maximum entropy method using piecewise linear, piecewise quadratic, and piecewise cubic splines.

Example 3.1. We consider the well known logistic map $\tau:[0,1] \rightarrow[0,1]$ defined by $\tau(x)=4 x(1-x)$. The actual density $f^{*}$ of $\tau$ is given by $f^{*}(x)=\frac{1}{\pi \sqrt{x(1-x)}}$. The logistic map $\tau$ is topologically conjugate to the tent map and it is known that the Lyapunov exponent for the logistic map $\tau$ is $l=\ln 2=0.693147$. Now, we apply (a) piecewise linear maximum entropy method; (b) piecewise quadratic maximum entropy method; (c) piecewise cubic maximum entropy method and we compute approximate Lyapunov exponent $l_{n}$, error $E_{n}=\left|l_{n}-l\right|$, the approximate density function $f_{n}$ and the $L^{1}-$ norm $\left\|f_{n}-f^{*}\right\|_{1}$.

In Figure 3.1, using (a) piecewise linear maximum entropy method; (b) piecewise quadratic maximum entropy method and (c) piecewise cubic maximum entropy method, we present the graph of the actual density function $f^{*}(x)=\frac{1}{\pi \sqrt{x(1-x)}}($ red $)$ and the graph of the approximate density function $f_{n}$ (blue). Gauss quadrature method is used for integrations. 500,000 iterations are used for the approximation of moments.

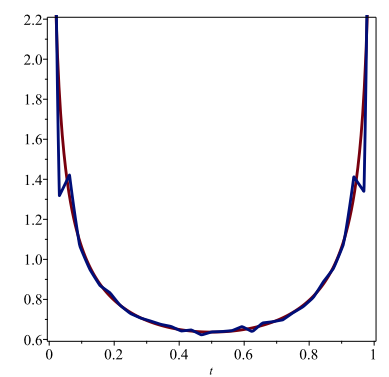

(a) A subfigure

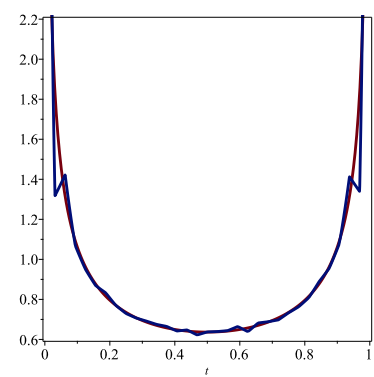

(b) A subfigure

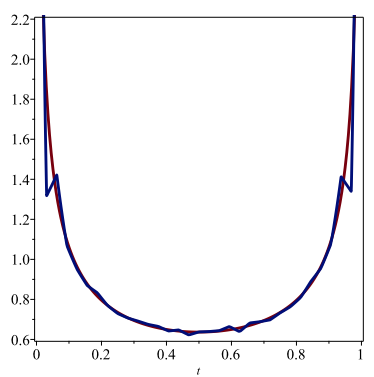

(c) A subfigure

Figure 3.1: This is lot of figures arranged side by side in matrix form with captions for each and a main caption

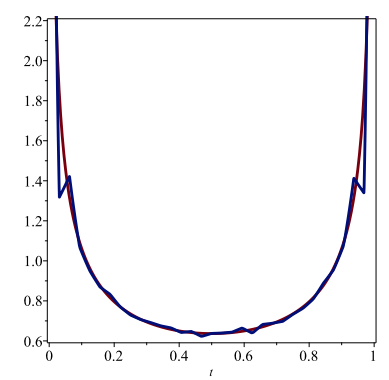

(a)

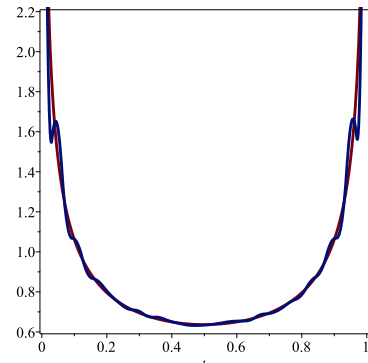

(b)

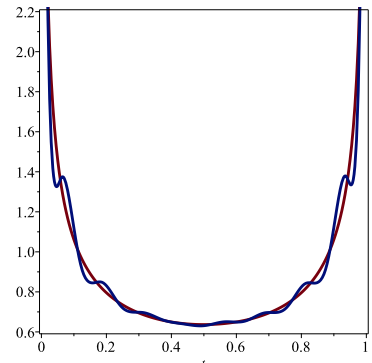

(c)

Figure 3.2: Approximate density $f_{n}$ of the density function $f^{*}$ of invariant measure $\mu^{*}$ for the map $\tau$ via piecewise spline maximum entropy method: Figure 3.1 (a) approximate density $f_{32}$ (blue) via piecewise linear maximum entropy method and the actual density $f^{*}$ (red); Figure 3.1 (b) approximate density $f_{32}$ (blue) via piecewise quadratic maximum entropy method and the actual density $f^{*}$ (red); Figure 3.1 (c) approximate density $f_{16}$ (blue) via piecewise cubic maximum entropy method and the actual density $f^{*}$ (red). 


\begin{tabular}{|c|c|c|c|}
\hline$n$ & $l_{n}$ (piecewise linear) & $\left|l_{n}-l\right|$ (piecewise linear) & $\left\|f_{n}-f^{*}\right\|_{1}$ (piecewise linear) \\
\hline 4 & 0.68775 & 0.00539 & 0.22098 \\
\hline 8 & 0.69363 & 0.00049 & 0.15821 \\
\hline 16 & 0.69355 & 0.00040 & 0.10494 \\
\hline 32 & 0.69361 & 0.00046 & 0.07745 \\
\hline
\end{tabular}

\begin{tabular}{|c|c|c|c|}
\hline$n$ & $l_{n}$ (piecewise quad) & $\left|l_{n}-l\right|$ (piecewise quad) & $\left\|f_{n}-f^{*}\right\|_{1}$ (piecewise quad) \\
\hline 4 & 0.67348 & 0.01965 & 0.17346 \\
\hline 8 & 0.69092 & 0.00222 & 0.12631 \\
\hline 16 & 0.693443 & 0.0002 & 0.08778 \\
\hline 32 & 0.69352 & 0.00037 & 0.05108 \\
\hline
\end{tabular}

\begin{tabular}{|c|c|c|c|}
\hline$n$ & $l_{n}$ (piecewise cubic) & (piecewise cubic) & $\left\|f_{n}-f^{*}\right\|_{1}$ (piecewise cubic) \\
\hline 4 & 0.70605 & 0.01290 & 0.15001 \\
\hline 8 & 0.69570 & 0.00255 & 0.10621 \\
\hline 16 & 0.69391 & 0.00255 & 0.06343 \\
\hline 32 & 0.69352 & 0.00037 & 0.05108 \\
\hline
\end{tabular}

The density function $f^{*}(x)=\frac{1}{\pi \sqrt{x(1-x)}}$ of the invariant measure $\mu^{*}$ is known for the logistic map $\tau(x)=4 x(1-x)$. Therefore, instead of (2.7) one can use (2.4) for the calculations of moments. In the following tables we present some approximate Lyapunov exponents $l_{n}$ and error $\left|l_{n}-l\right|$ using piecewise linear, piecewise quadratic and piecewise cubic maximum entropy method, where we have used (2.4) for moments.

\begin{tabular}{|c|c|c|c|}
\hline piecewise spline method & $n$ & $l_{n}$ & $\left|l_{n}-l\right|$ \\
\hline piecewise linear & 4 & 0.68812 & 0.00503 \\
\hline piecewise linear & 32 & 0.69363 & 0.00048 \\
\hline piecewise quad & 32 & 0.69349 & 0.00034 \\
\hline piecewise cubic & 32 & 0.69352 & 0.00037 \\
\hline
\end{tabular}

Example 3.2. We consider the map $\tau:[0,1] \rightarrow[0,1]$ defined by

$$
\tau(x)= \begin{cases}\frac{3}{2} x^{2}+\frac{5}{4} x, & 0 \leq x<\frac{1}{2}, \\ \frac{3}{2}(1-x)^{2}+\frac{5}{4}(1-x), & \frac{1}{2} \leq x \leq 1,\end{cases}
$$

The map $\tau$ is is a piecewise expanding map. However, analytical density function of $\tau$ is not known. We apply (a) piecewise linear maximum entropy method; (b) piecewise quadratic maximum entropy method; (c) piecewise cubic maximum entropy method and we compute approximate Lyapunov exponent $l_{n}$.

\begin{tabular}{|c|c|c|c|}
\hline$n$ & $l_{n}$ (piecewise linear) & $l_{n}$ (piecewise quadratic) & $l_{n}$ (piecewise cubic) \\
\hline 4 & 0.63118 & 0.63104 & 0.63132 \\
\hline 8 & 0.63105 & 0.63104 & 0.63114 \\
\hline 16 & 0.63105 & 0.63104 & 0.63105 \\
\hline
\end{tabular}

In Figure 3.2, using (a) piecewise linear maximum entropy method; (b) piecewise quadratic maximum entropy method and (c) piecewise cubic maximum entropy method, we present the graph of the histogram (with 500,000 iterations) of an approximate density function (black) and the graph of the approximate density function $f_{n}(r e d)$. Gauss quadrature method is used for integrations.

Example 3.3. We consider the map $\tau:[0,1] \rightarrow[0,1]$ defined by

$$
\tau(x)= \begin{cases}\frac{2 x}{1-x}, & 0 \leq x<\frac{1}{3}, \\ \frac{1-x}{2 x}, & \frac{1}{3} \leq x \leq 1,\end{cases}
$$

The actual density $f^{*}$ of $\tau$ is given by $f^{*}(x)=\frac{2}{(1-x)^{2}}$. The Lyapunov exponent for the map $\tau$ is $l \approx=0.693147$. Now, we apply (a) piecewise linear maximum entropy method; (b) piecewise quadratic maximum entropy method; (c) piecewise cubic maximum entropy method and we compute approximate Lyapunov exponent $l_{n}$, error $E_{n}=\left|l_{n}-l\right|$.

In Figure 3.3, using (a) piecewise linear maximum entropy method; (b) piecewise quadratic maximum entropy method and (c) piecewise cubic maximum entropy method, we present the graph of the actual density function $f^{*}(x)=\frac{2}{(1-x)^{2}}$. (red) and the graph of the approximate density function $f_{n}$ (blue). Gauss quadrature method is used for integrations. 500,000 iterations are used for the approximation of moments. 


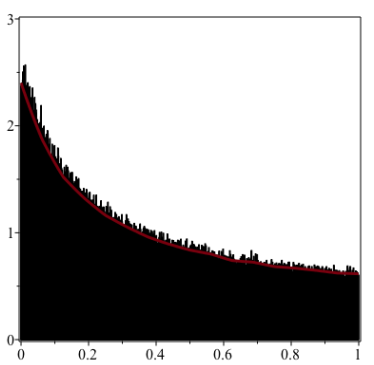

(a)

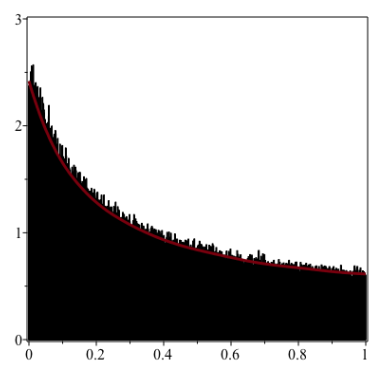

(b)

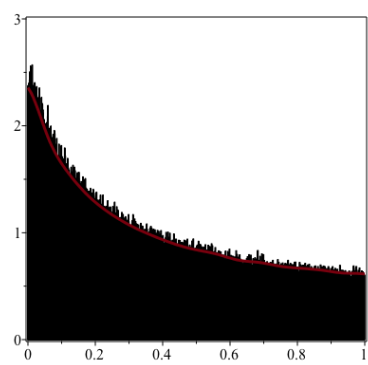

(c)

Figure 3.3: Histogram and approximate density via piecewise spline maximum entropy method: Figure 3.2 (a) the histogram of the density function of the map $\tau$ with 500,000 points on the trajectory of the map $\tau$ with 1000 subintervals for $[0,1]$. and the graph of the approximate density function $f_{16}$ via piecewise linear maximum entropy method; Figure 3.2 (b) the same histogram and the graph of the approximate density function $f_{8}$ via piecewise quadratic maximum entropy method; Figure 3.2 (c) the same histogram and the graph of the approximate density function $f_{16}$ via piecewise cubic maximum entropy method;

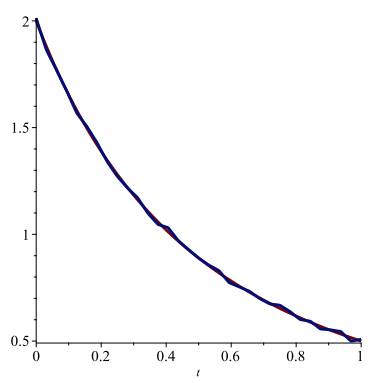

(a)

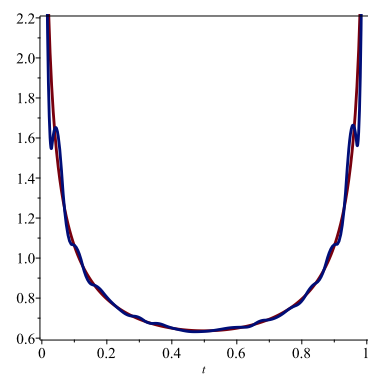

(b)

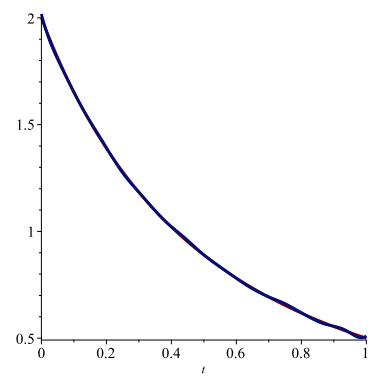

(c)

Figure 3.4: Approximate density $f_{n}$ of the density function $f^{*}$ of invariant measure $\mu^{*}$ for the map $\tau$ via piecewise spline maximum entropy method: Figure 3.3 (a) approximate density $f_{32}$ (blue) via piecewise linear maximum entropy method and the actual density $f^{*}$ (red); Figure 3.2 (b) approximate density $f_{32}$ (blue) via piecewise quadratic maximum entropy method and the actual density $f^{*}$ (red); Figure 3.3 (c) approximate density $f_{16}$ (blue) via piecewise cubic maximum entropy method and the actual density $f^{*}$ (red).

\begin{tabular}{|c|c|c|}
\hline$n$ & $l_{n}$ (piecewise linear) & $\left|l_{n}-l\right|$ (piecewise linear) \\
\hline 4 & 0.69323 & 0.00009 \\
\hline 8 & 0.69316 & 0.00001 \\
\hline 16 & 0.69316 & 0.00001 \\
\hline 32 & 0.69314 & 0.0000008 \\
\hline
\end{tabular}

\begin{tabular}{|c|c|c|}
\hline$n$ & $l_{n}$ (piecewise quad) & $\left|l_{n}-l\right|$ (piecewise quad) \\
\hline 4 & 0.69323 & 0.00008 \\
\hline 8 & 0.69314 & 0.00005 \\
\hline 16 & 0.69313 & 0.00001 \\
\hline 32 & 0.69314 & 0.000001 \\
\hline
\end{tabular}

\begin{tabular}{|c|c|c|}
\hline$n$ & $l_{n}$ (piecewise cubic) & (piecewise cubic) \\
\hline 4 & 0.69300 & 0.00014 \\
\hline 8 & 0.69325 & 0.00010 \\
\hline 16 & 0.69314 & 0.00003 \\
\hline
\end{tabular}

The density function $f^{*}(x)=\frac{2}{(1-x)^{2}}$ of the invariant measure $\mu^{*}$ is known for the map $\tau$. Therefore, instead of (2.7) one can use (2.4) for

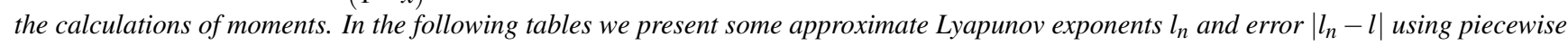
linear and piecewise quadratic maximum entropy method, where we have used (2.4) for moments. 


\begin{tabular}{|c|c|c|c|}
\hline piecewise spline method & $n$ & $l_{n}$ & $\left|l_{n}-l\right|$ \\
\hline piecewise linear & 4 & 0.69324 & 0.00008 \\
\hline piecewise linear & 16 & 0.69314 & 0.000005 \\
\hline piecewise quad & 16 & 0.69314 & 0.000005 \\
\hline
\end{tabular}

\section{Conclusion}

In this paper, we study numerical computations of Lyapunov exponents for deterministic chaotic dynamical systems in one dimension. First, we discuss the fact that the Lyapunov exponent is one of the key tools for determining whether a deterministic dynamical system is chaotic or not. Moreover, we show that the computation of Lyapunov exponents of dynamical systems depends on the computation of invariant measures of dynamical systems. Then, we study a general piecewise spline maximum entropy method for the computation of Lyapunov exponents and invariant measures for deterministic dynamical systems. We present a proof of convergence of the general piecewise spline maximum entropy method. The general piecewise spline maximum entropy method includes piecewise linear, piecewise quadratic, piecewise cubic and higher order maximum entropy methods. Finally, we present two examples where we compute Lyapunov exponents of dynamical systems via piecewise linear, piecewise quadratic and piecewise cubic maximum entropy methods. Moreover, we compute invariant measures of the dynamical systems. In the first example we present errors between the numerical results and analytical results (both for Lyapunov exponent and invariant measures). The numerical examples show that the piecewise spline maximum entropy method is a useful method for the computation of Lyapunov exponents and invariant measures for deterministic dynamical systems. In future we plan to study the speed of convergence of the piecewise spline maximum entropy method for the computation of Lyapunov exponents and invariant measures.

\section{Acknowledgement}

The author is grateful to anonymous referees for their valuable comments which improves the presentation of the manuscript. This research is funded by NSERC DG grant 2017-05321 of the author at the University of Prince Edward Island, PE, Canada.

\section{References}

[1] A. M. Lyapunov, Problem Général de la Stabilité du Mouvement (French), Ann. Fac. Univ. Touluse, 9(2) (1947), $203-474$.

[2] V. I. Oseledec, A multiplicative ergodic theorem. Characteristic Liapunov, exponents of dynamical systems (Russian), Tr. Mosk. Mat. Obs., 19 (1968), 79-210.

[3] A. Wolf, Quantifying chaos with Lyapunov exponents, Nonlinear Sci. Theory Appl., Manchester Univ. Press, Manchester, 1986.

[4] A. Boyarsky, A matrix method for estimating the Lyapunov exponent of one-dimensional systems, J. Stat. Phys., 50(1-2) (1988), $213-229$.

[5] C. Robinson, Dynamical Systems : Stability, Symbolic Dynamics, and Chaos, Boca Raton : CRC Press, 1995.

[6] A. Lasota, M. C. Mackey, Chaos, Fractals, and Noise. Stochastic Aspects of Dynamics, Second edition, Springer-Verlag, New York, 1994.

[7] G. Froyland, K. Judd, A. I. Mess, Estimation of dynamical systems using a spatial average, Phys. Rev. E (3), 51(4 part A) (1995), 2844-2855 .

[8] G. Gencaya, W. D. Dechert, An algorithm for the $n$ Lyapunov exponents of an n-dimensional unknown dynamical system, Phys. D: Nonlinear Phenom., 59(1-3) (1992), 142-157.

[9] P. Bryant, R. Brown, H. D. I. Abarbenel, Lyapunov exponents from observed time series, Phys. Rev. Lett., 65(13) (1990), 1523-1526.

[10] S. Ellner, A. R. Gallant, D. McCaffrey, D. Nychka, Convergence rates and data requirements for Jacobian based estimates of Lyapunov exponents from data, Physics Letters A, 153 (6-7) (1991), 357-363.

[11] L. R. Mead, N. Papanicolaou, Maximum entropy in the problem of moments, J. Math. Phys., 25 (1984), 2404 - -2417.

[12] E. T. Jaynes, Information theory and statistical mechanics, Phys. Rev., 106 (1957), 620-630.

[13] J. Ding, N. H. Rhee, A unified maximum entropy method via spline functions for Frobenius -Perron operators, Numer. Algebra Control Optim., 3(2) (2013), 235-245.

[14] J. Ding, A maximum entropy method for solving Frobenius-Perron equations, Appl. Math. Comp., 93 (1998), 155-168.

[15] J. Ding, C. Jin, N. H. Rhee, A. Zhou, A maximum entropy method based on piecewise linear functions for the recovery of a stationary density of interval maps, J. Stat. Phys., 145 (2011), 1620-1639.

[16] J. Ding, R. L. Mead, The maximum entropy method applied to stationary density computation, Appl. Math. Comp., 185 (2007), 658-666.

[17] J. Ding, N. H. Rhee, A maximum entropy method based on orthogonal polynomials for Frobenius-Perron operators, Adv. Applied Math. Mec., 3(2) (2011), 204-218.

[18] J. Ding, N. H. Rhee, Birkhoff's ergodic theorem and the piecewise constant maximum entropy method for Frobenius-Perron operators, Inter. J. Computer Math., 89(8) (2012), 1083-1091.

[19] T. Upadhay, J. Ding, N. H. Rhee, A piecewise quadratic maximum entropy method for the statistical study of chaos, J. Math. Anal. Appl., 421 (2015),

[20] C. J. Bose, R. Murray, Dynamical conditions for convergence of a maximum entropy method for Frobenius-Perron operator equations., Appl. Math. Comput., 182(1) (2006), 210-212.

[21] M. S. Islam, Maximum entropy method for position dependent random maps, Internat. J. Bifur. Chaos Appl. Sci. Engng., 21 (2011), $1805-1811$.

[22] M. S. Islam, A piecewise quadratic maximum entropy method for invariant measures of position dependent random maps, Dyn. Contin. Discrete Impuls. Syst. Ser. A Math. Anal., 24(6) (2017), 431-445.

[23] P. Biswas, H. Shimoyama, R. L. Mead, Lyapunov exponents and the natural invariant density determination of chaotic maps: an iterative maximum entropy ansatz, J. Phys. A: Math. Theor., 43(12) (2010), 1-12.

[24] A. Lasota, J. A. Yorke, On the existence of invariant measures for piecewise monotonic transformations, Trans. Amer. Math. Soc., 186 (1973), $481-488$.

[25] J. M. Borwein, A. S. Lewis, Convergence of the best entropy estimates, SIAM J. Optim., 1(2) (1991), $191-205$. 